\title{
Cluster-Based Test Scheduling Strategies Using Semantic Relationships between Test Specifications
}

\author{
Sahar Tahvili \\ RISE SICS Västerås $\mathrm{AB}$ \\ Västerås, Sweden \\ sahar.tahvili@ri.se \\ Wasif Afzal \\ Mälardalen University \\ Västerås, Sweden \\ wasif.afzal@mdh.se
}

\author{
Leo Hatvani \\ Mälardalen University \\ Västerås, Sweden \\ leo.hatvani@mdh.se \\ Mehrdad Saadatmand \\ RISE SICS Västerås AB \\ Västerås, Sweden \\ mehrdad.saadatmand@ri.se
}

\author{
Michael Felderer \\ University of Innsbruck \\ Innsbruck, Austria \\ michael.felderer@uibk.ac.at \\ Markus Bohlin \\ RISE SICS Västerås $A B$ \\ Västerås, Sweden \\ markus.bohlin@ri.se
}

\begin{abstract}
One of the challenging issues in improving the test efficiency is that of achieving a balance between testing goals and testing resources. Test execution scheduling is one way of saving time and budget, where a set of test cases are grouped and tested at the same time. To have an optimal test execution schedule, all related information of a test case (e.g. execution time, functionality to be tested, dependency and similarity with other test cases) need to be analyzed. Test scheduling problem becomes more complicated at high-level testing, such as integration testing and especially in manual testing procedure. Test specifications are generally written in natural text by humans and usually contain ambiguity and uncertainty. Therefore, analyzing a test specification demands a strong learning algorithm. In this position paper, we propose a natural language processing-based approach that, given test specifications at the integration level, allows automatic detection of test cases semantic dependencies. The proposed approach utilizes the Doc2Vec algorithm and converts each test case into a vector in n-dimensional space. These vectors are then grouped using the HDBSCAN clustering algorithm into semantic clusters. Finally, a set of cluster-based test scheduling strategies are proposed for execution. The proposed approach has been applied in a sub-system from the railway domain by analyzing an ongoing testing project at Bombardier Transportation AB, Sweden.
\end{abstract}

\section{KEYWORDS}

Software testing, Test optimization, NLP, Dependency, Clustering, Doc2Vec, HDBSCAN

ACM Reference Format:

Sahar Tahvili, Leo Hatvani, Michael Felderer, Wasif Afzal, Mehrdad Saadatmand, and Markus Bohlin. 2018. Cluster-Based Test Scheduling Strategies Using Semantic Relationships between Test Specifications. In RET'18: RET'18:IEEE/ACM 5th International Workshop on Requirements Engineering

Permission to make digital or hard copies of all or part of this work for personal or classroom use is granted without fee provided that copies are not made or distributed for profit or commercial advantage and that copies bear this notice and the full citation on the first page. Copyrights for components of this work owned by others than ACM must be honored. Abstracting with credit is permitted. To copy otherwise, or republish, to post on servers or to redistribute to lists, requires prior specific permission and/or a fee. Request permissions from permissions@acm.org.

RET'18, June 2, 2018, Gothenburg, Sweden

(C) 2018 Association for Computing Machinery.

ACM ISBN 978-1-4503-5749-4/18/06...\$15.00

https://doi.org/10.1145/3195538.3195540 and Testing, June 2, 2018, Gothenburg, Sweden. ACM, New York, NY, USA, 4 pages. https://doi.org/10.1145/3195538.3195540

\section{INTRODUCTION}

For over a decade, applications of natural language processing (NLP) techniques were considered in different domains such as machine learning, deep learning, artificial intelligence, and static analysis. Moreover, using the NLP techniques might be a suitable approach for improving the efficiency of documentation processes, which can be applied in different phases of software development life cycle (SDLC) such as requirement analysis, planning, and also testing Since, in many cases, both requirements and test cases are formulated in a natural text, NLP techniques can be used for analyzing the details of a textual specification. Some specific information such as the functionality to be tested and the relationship between test cases can be detected by analyzing the textual specifications. One challenge for improving a testing process is detecting the dependency between test cases. By having an overview of dependent test cases, we can prioritize, select, and schedule test cases for execution. Some previous studies show that, there are different types of dependency between test cases, which will impact the result of a test execution [2]. In our previous work [15] we proposed a manual method for measuring the degree of dependency between test cases. Since the number of required test cases for testing a system is rather large, a manual approach is not suitable to solve the problem efficiently. In the present position paper, we propose an NLP-based approach for detecting the semantic dependency between test cases automatically, based on the textual test specifications. We also present a set of cluster-based strategies for scheduling tests. This paper provides the following contributions: (1) detecting the semantic dependency between test cases through analyzing test specifications, (2) clustering test cases based on the semantic dependency, and (3) proposing a set of cluster-based test scheduling strategies for execution.

\section{BACKGROUND AND RELATED WORK}

The lack of accurate test specifications analysis may lead to an inefficient testing process. Knowing the execution time, requirement coverage and the dependency between test cases, are some required information that testers have to receive in the early stage of a testing process. Paying no attention to dependencies between test cases may lead to sequential failure of them and thereby waste of testing resources. In [13] we showed that the dependencies among test 


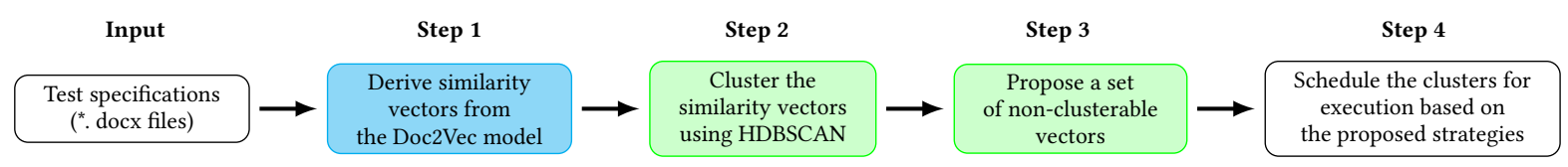

Figure 1: The steps of the proposed approach

cases give partial information on the verdict of a test case from the verdict of another one. Arlt et al. in [2] showed that test cases will be failed after each other if the testers do not care about the dependency between test cases. In integration testing, the software modules are combined and tested as a group, therefore, the problem of detecting the dependency between test cases will be more highlighted for the testers. Test optimization is the main purpose of using test case dependency information, where test cases will be prioritized and scheduled by using this. Indumathi et al. in [1] propose a manual method for detecting the functional dependency between test cases. The proposed method in [1] deals with the sequence execution between test cases. Since the dependency information is extracted manually, the method is not scalable to handle a large set of test cases. Component dependency model (CMD) represents another type of test cases dependency, introduced by Caliebe et al. in [4]. The proposed method in [4] is applicable for the componentbased systems and the dependencies can be identified through analyzing system structure, architecture, and requirements.

\section{SEMANTIC-DEPENDENCY MODEL}

None of the researched dependencies in software testing domain, handles the semantic relationships between the test cases. Generally, the semantic relationship is the associations that exist between the meanings of sentences [12]. The semantic dependency between manual test cases can be summarized as the conceptual associations between the meaning of the test specifications. A typical test specification has multiple test steps including preconditions, test activities and post-conditions, which are described textually. Therefore, detecting the semantic relationship between test cases, which are conceptually associated, requires deeper vocabulary analysis. Executing a group of test cases, which are semantically dependent on each other, can lead to an optimal usage of testing resources. In following scenarios are considered in the present work:

- Test cases have similar preconditions, initial state or postconditions

A precondition for a test case specifies the setup needed for the test case to be executed successfully. The precondition includes the state that a system and its environment must satisfy before executing a test case [3]. A post-condition is a statement which describes the conditions that will be true when a test case has been executed successfully. The test specifications are written by humans and are not easy to detect the similar preconditions and neither the post-conditions. Moreover, making a system ready for testing (running the preconditions in a test case) is a resource consuming process. Therefore, when the system is reaching an acceptable state, more test cases, which require the same system state, can be tested as a group together. However, though running those test cases which require same post-conditions, we are able to save more time.

\section{- Test cases test same functionality of a software}

The main function which will be tested by a manual test case, is usually described textually into several test steps. Moreover, a functionality of a software to be tested, can be divided within different test cases. Providing a group of test cases which test the same functionality, can help testers to save more time. Test cases which have a semantic dependency might test same functionality of a software application and should be tested in succession.

\section{THE APPROACH}

This section describes our approach for scheduling manual test cases based on the semantic dependency between them. The proposed approach is based on the valuation of test specifications, as well as clustering of the dependent test cases. Three test scheduling strategies, based on the semantic dependency between the test cases, will be proposed. Our approach is based on two main algorithms, which can handle a large set of data. Moreover, the testers can easily re-run the proposed algorithms, when new test specifications are added to the set. Figure 1 represents the structure of our approach, where the blue color represents the NLP stage and the green color shows the cluster analysis stages. The steps of the proposed approach are described in follow:

Step 1: detecting the semantic relationships between manual test cases using the Doc2Vec algorithm.

Step 2: cluster the semantic dependent test cases using HDBSCAN algorithm.

Step 3: propose a set of non-clusterable test cases as independent test cases.

Step 4: use one of the cluster-based scheduling strategies for test execution.

As illustrated in Figure 1, the required input for running our approach is a test specification. By running the Doc2Vec algorithm, a set of vectors (which represent each test case) in a $n$-dimensional space will be generated. Thereby, HDBSCAN algorithm classifies test cases into several clusters. Finally, a set of scheduling strategies will be proposed for execution. In other words, the expected output of the proposed approach is a set of semantic dependent test cases, arranged for execution, based on scheduling strategies.

\subsection{Document Embedding Using Doc2Vec Algorithm}

The Doc2Vec algorithm consists a set of shallow and two-layer neural networks models which are designed to produce document embedding [10]. The basis of the Doc2Vec is based on learning representations forward in such a way that Doc2Vec takes as its input a large corpus of text and generates a unique vector in $n$-dimensional space for each unique document in the corpus [8]. From here, we can measure the similarity between two documents by calculating 
the Cosine similarity of the two vectors that represent the corresponding documents. The similarities in the $n$-dimensional space can be extracted to do comparison [8] where the words with similar meaning end up lying close to each other. Furthermore, the Doc2Vec uses vector arithmetic to work with analogies, for instance the famous example: Doctor - Man + Woman = Nurse and also USB - Port + Display = HDMI. In this study, we train the Doc2Vec algorithm in such a way that each test specification is considered as an input document. As the first step, Doc2Vec extracts the semantic dependency between test specifications automatically, through utilizing deep linguistic patterns which have been defined over the dependency grammar of sentences [8].

\subsection{Clustering with HDBSCAN Algorithm}

After running the Doc2Vec algorithm, a set of high dimensional vectors (which represents each test specification) is generated. The vectors which have less distances to each other can be classified as a cluster. This step, will be performed by a fast and robust algorithm called HDBSCAN (Hierarchical Density-Based Spatial Clustering of Applications with Noise), which handles large high-dimensional data sets. The HDBSCAN measures the distance between the vectors and provides a set of clusters and also a set of non-clusterable vectors. Other clustering algorithms are designed to cluster every vector to some cluster, which indicates their inability to handle noise in the clustering process, or, in some case, the algorithms cannot properly process high-dimensional data. In this work, we interpret the non-clusterable vectors as independent test cases, which can be executed in no particular order. Furthermore, each cluster consists of a set of test cases which have a semantic dependency and must be tested together at the same time.

\subsection{Cluster-Based Test Scheduling Strategies}

In this subsection, we propose a set of cluster-based test case scheduling strategies based on the semantic dependency between test cases. The following definitions are applicable:

Definition 4.1. Let $C:=\left\{C_{1}, C_{2}, \ldots, C_{n}\right\}$ where, each $C_{j}$ is a cluster, $j=1,2, \ldots, n$.

Definition 4.2. Let cardinality of each $C_{j}$ be $K_{j}$, where $K_{j} \in \mathbb{N}$ and $K_{j}>1$. We define $K_{j}$ as a cluster size. The size of cluster represents the number of test cases per cluster.

Definition 4.3. $\mathcal{P}:=\left\{P_{1}, P_{2}, \ldots, P_{m}\right\}$ where each $P_{j}$ is a unique property for all member of $C_{j}$; that is $\forall x \in C_{j}, \exists ! P_{s_{j}}$ such that $x \in P_{s_{j}}$, for every $j \in\{1,2, . ., n\}$.

In this work, we utilize the functional requirement group (FG) as a cluster property, where, every test case belongs to one functional group and tests a specific part of a system under test. For instance, brake system and radio are two different functional groups.

Definition 4.4. We define $t_{j}$ as a time function for every $C_{j}$ such that $t_{j}: C_{j} \longrightarrow(0, \infty)$.

The time function in the present work, represents the required time that a cluster of test cases takes for execution (the sum of test cases execution time). Since each test case takes different time for execution, then $t_{j}$ will be changed for every $C_{j}$. In [14], we showed that the execution time for test cases can be predicted by performing some regression analysis on previous executed test cases. If there is no execution data available for test cases, we can assume all test cases have the same execution time. Using the proposed definitions, we define the following cluster-based strategies for test case scheduling:

- Strategy $1: C_{i}$ has a higher priority than $C_{j}$ if and only if $K_{i}>K_{j}$, where $i, j \in\{1,2, \ldots, n\}$, moreover this strategy can be called as increasingly ordering. However, strategy 1 can be defined as decreasingly ordering such that: $C_{i}$ has a higher priority than $C_{j}$ if and only if $K_{i}<K_{j}$, where $i, j \in\{1,2, \ldots, n\}$.

In other words, the clusters are ranked in strategy 1 based on their size. However, if two (or more) clusters have a same size $\left(K_{i}=K_{j}\right)$, then strategy 1 is not applicable and a new strategy should be considered.

- Strategy 2 : Let $C_{i}=\left\{x_{1, i}, x_{2, i}, \ldots, x_{K_{i}, i}\right\}, C_{j}=\left\{x_{1, j}, x_{2, j}, \ldots, x_{K_{j}, j}\right\}$, where $K_{i}=K_{j}$. If $x_{l, i} \in P_{s_{l}, i}$ and $x_{l, j} \in P_{s_{l}, j}$, for $l=1,2, \ldots, K_{i}$. We define: $\mathcal{I}:=\left\{P_{s_{1}, i}, P_{s_{2}, i}, \ldots, P_{s_{K_{i}}}, i\right\}$ and $\mathcal{J}:=\left\{P_{s_{1}, j}, P_{s_{2}, j}, \ldots, P_{s_{K_{j}}, j}\right\}$. Thus $C_{i}$ has a higher priority than $C_{j}$ if and only if $|\mathcal{I}|>|\mathcal{J}|$.

In strategy 2 , the clusters which contain test case with different property (functional group) are top ranked. In fact, if $C_{i}$ includes two test cases with two different properties ( 2 unique FGs), has a higher priority than $C_{j}$ with the same size (2 test cases) which test just one FG (test cases have a same property). However, strategy 2 is not applicable if two (or more) clusters contain test cases which have a same property $(|\mathcal{I}|=|\mathcal{J}|)$ with the same size $\left(K_{i}=K_{j}\right)$.

- Strategy 3 : We define $T_{i}:=\sum_{k=1}^{K_{i}} t_{i}\left(x_{k, i}\right), x_{k, i} \in C_{i}$ and $T_{j}:=\sum_{k=1}^{K_{j}} t_{j}\left(x_{k, j}\right), x_{k, j} \in C_{j}$, where $K_{i}=K_{j}$ and $k \in\left\{1,2, \ldots, K_{i}\right\}$. Thus $C_{i}$ has a higher priority than $C_{j}$ if and only if $T_{i}<T_{j}$.

However if $T_{i}=T_{j}$, there is no priority ordering to those clusters. In strategy 3 , the clusters which take less time for execution are top ranked. Strategy 3 is applicable when an estimation of execution time for test cases is available. As stated before, through historical analysis of previously executed test cases, the execution time for test cases can be predicted [14]. Furthermore, if we assume same execution time for test cases we are faced with the situation that two (or more) clusters have a same size and same property, we can run those clusters without any order.

\section{PROOF OF CONCEPT}

The feasibility of the proposed approach has been done through studying an on-going project for underground subway train in Stockholm, called C30 project at Bombardier Transportation AB.

\subsection{Case Study Report}

The units of analysis in this case study are test specifications at the integration testing level for $C 30$ project. A total of 29 test suites, which are designed for testing different functional groups, have been extracted from the DOORS database at Bombardier. The extracted test cases are converted into vectors using paragraphvectors [5] implementation of the Doc2Vec model, and then the test cases are clustered by applying HDBSCAN [6] algorithm. Table 1 lists the functional groups and the number of associated test cases for testing various FG. Moreover, these test cases are run at a sub-system level, meaning that they are more time consuming 
to run than tests at unit level [7]. Therefore, executing dependent test cases which require same setup and conditions can help testers significantly reduce the testing time.

\begin{tabular}{c|l|c|c|c}
\hline No. & Functional group & Test Case & Steps & Words \\
\hline 1 & ATP & 6 & 35 & 458 \\
2 & Air Supply & 2 & 19 & 844 \\
3 & Bogie & 4 & 29 & 779 \\
4 & Brake system & 7 & 28 & 476 \\
5 & DVS & 1 & 5 & 141 \\
$\ldots$ & $\ldots$ & $\ldots$ & $\ldots$ & $\ldots$ \\
28 & Vehicle Coupler & 11 & 40 & 488 \\
29 & Windscreen & 4 & 34 & 345 \\
\hline
\end{tabular}

Table 1: Functional group with associated test cases (partial)

Moreover, Table 2 represents the clusters of test cases which are generated by the HDBSCAN algorithm.

\begin{tabular}{c|c|l}
\hline Cluster & Test Case & Functional Groups \\
\hline$C_{u}$ & 126 & Independent test cases \\
$C_{1}$ & 6 & Drive and Brake Function, DVS, ATP, Traffic Radio Functions \\
$C_{2}$ & 4 & Drive and Brake Function, Safe Exterior Access, CCTV \\
$C_{3}$ & 9 & Safe Exterior Access, Train Inauguration \\
$C_{4}$ & 3 & Safe SITS \\
$C_{5}$ & 7 & Safe Bogie, General Requirements \\
$C_{6}$ & 8 & Train Inauguration Functions, Drive and Brake Function \\
$C_{7}$ & 6 & Exterior and Interior Access Function, Safe SITS \\
$\ldots$ & $\ldots$ & $\ldots$ \\
\hline
\end{tabular}

Table 2: The proposed clusters for scheduling (partial)

In Table 2, 126 test cases are identified as independent test cases. A total of 93 clusters are determined for dependent test cases (not all clusters are shown in Table 2). Test cases in Table 2 are created for integration testing, where individual FGs will be combined and tested as a group, moreover, most of the generated clusters in Table 2 are included test cases from different FGs. Some clusters such as $C_{4}$ contain test cases from just one FG. In this study, we just rank some of the generated clusters in Table 2 according to the proposed strategies in Section 4. Table 3 represents the number of 7 clusters which are ranked based on three different strategies. Since the execution time for each test case is predictable for us [14], thereby, strategy 3 is applicable in this work. As Table 3 shows, the execution time for 7 tests cases in cluster $C_{5}$ is less than 3 test cases in cluster $C_{4}$, therefore $C_{5}$ has a higher priority than $C_{4}$. However, independent test cases have been clustered in $C_{u}$ which can be executed without any particular order or other criteria such as execution time and requirement coverage can be considered for ranking them.

\begin{tabular}{l|l}
\hline Strategy & Cluster \\
\hline Strategy 1 & $C_{3}, C_{6}, C_{5}, C_{7}, C_{1}, C_{2}, C_{4}$ \\
\hline Strategy 2 & $C_{1}, C_{2}, C_{3}, C_{5}, C_{6}, C_{7}, C_{4}$ \\
\hline Strategy 3 & $C_{5}, C_{4}, C_{6}, C_{1}, C_{2}, C_{7}, C_{3}$
\end{tabular}

Table 3: The proposed scheduling strategies

\section{DISCUSSION \& FUTURE EXTENSIONS}

To detect different type of dependencies between test cases, various phases of a software development life cycle (SDLC) such as design, requirements and testing need to be analyzed. In this paper, we strive to propose a solution for such a situation that other information (requirement, internal signals, software architecture, etc.) are assumed to be not available. Executing a set of test cases which require the same system and environment setting is extractable from test specification. However, other clustering algorithms such as Fuzzy C-means, k-means can be utilized for the clustering part of the proposed approach. Finally, a decision support system can be designed to schedule test cases for execution, based on the dependencies between test cases, requirement coverage and execution time.

\section{SUMMARY \& CONCLUSION}

In this position paper, we proposed a cluster-based approach for scheduling integration test cases based on the semantic dependency between test specifications. The proposed approach has been applied to an industrial use case at Bombardier Transportation AB. The results of the proof of concept indicate that the concept of semantic dependency exists between integration test cases from different functional groups and can be detected through text analysis. Additionally, test cases have been divided into several clusters based on their semantic dependencies. Moreover, three different scheduling strategies have been proposed, where test cases will be given a different order for execution based on the proposed strategies. Finally, we interpret non-clusterable vectors as independent test cases.

\section{REFERENCES}

[1] 2015. Test Cases Prioritization Using Open Dependency Structure Algorithm Procedia Computer Science 48 (2015), 250 - 255. International Conference on Computer, Communication and Convergence (ICCC 2015).

[2] S. Arlt, T. Morciniec, A. Podelski, and S. Wagner. 2015. If A Fails, Can B Still Succeed? Inferring Dependencies between Test Results in Automotive System Testing. In 8th International Conf. on Software Testing, Verification and Validation.

[3] M. Bertrand. 1997. Object-oriented Software Construction. Prentice-Hall, Inc.

[4] P. Caliebe, T. Herpel, and R. German. 2012. Dependency-Based Test Case Selection and Prioritization in Embedded Systems. In 5th International Conf. on Software Testing, Verification and Validation.

[5] L. Hatvani. 2018. Paragraph-vectors implementation. https://github.com/inejc/ paragraph-vectors. (2018).

[6] L. Hatvani. 2018. The used implementation of the HDBSCAN algorithm. https: //github.com/scikit-learn-contrib/hdbscan. (2018).

[7] H. Hemmati, L. Briand, A. Arcuri, and Sh. Ali. 2010. An enhanced test case selection approach for model-based testing: An industrial case study. In Proceedings of the 18th ACM SIGSOFT International Symposium on Foundations of Software Engineering. USA.

[8] Q. Le and T. Mikolov. 2014. Distributed Representations of Sentences and Documents. In Proceedings of the 31st International Conference on Machine Learning (Proceedings of Machine Learning Research), Vol. 32. China.

[9] I. Medeiros, N. Neves, and M. Correia. 2016. DEKANT: A Static Analysis Tool That Learns to Detect Web Application Vulnerabilities. In 25th International Symposium on Software Testing and Analysis (ISSTA 2016). USA.

[10] T. Mikolov, K. Chen, G. Corrado, and J. Dean. 2013. Efficient Estimation of Word Representations in Vector Space. CoRR abs/1301.3781 (2013). arXiv:1301.3781 http://arxiv.org/abs/1301.3781

[11] P. Runeson, , and H. Martin. 2008. Guidelines for conducting and reporting case study research in software engineering. Empirical Software Engineering 14, 2 (2008).

[12] V. C. Storey. 1993. Understanding semantic relationships. The VLDB fournal 2, 4 (01 Oct 1993), 455-488. https://doi.org/10.1007/BF01263048

[13] S. Tahvili, M. Bohlin, M. Saadatmand, S. Larsson, W. Afzal, and D. Sundmark. 2016 Cost-Benefit Analysis of Using Dependency Knowledge at Integration Testing. In 17th International Conf. On Product-Focused Software Process Improvement.

[14] S. Tahvili, M. Saadatmand, M. Bohlin, W. Afzal, and Sh. Hasan Ameerjan. 2017. Towards Execution Time Prediction for Test Cases from Test Specification. In 43rd Euromicro Conference on Software Engineering and Advanced Applications.

[15] S. Tahvili, M. Saadatmand, S. Larsson, W. Afzal, M. Bohlin, and D.Sundmark. 2016. Dynamic Integration Test Selection Based on Test Case Dependencies. In 11th Work. on Testing: Academia-Industry Collaboration, Practice and Research Techniques. 